\title{
Nachruf Hans Götschhofer
}

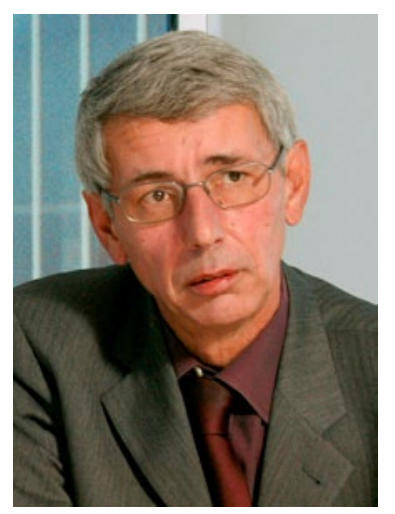

Am 30. Juni 2014 ist Herr Ing. Mag. Hans Götschhofer nach langer schwerer Krankheit im 67. Lebensjahr friedlich im Kreise seiner Familie, versehen mit den heiligen Sakramenten, für immer eingeschlafen.

Wir erinnern uns an einen immer aktiven, vielseitigen Konzernplaner der voestalpine AG, den Leiter der Unternehmensentwicklung der voestalpine Stahl $\mathrm{GmbH}$ und den Revisionsleiter der voestalpine AG.

In der langen Zeit der Zusammenarbeit haben wir Hans Götschhofer nicht nur als ausgezeichneten Betriebswirt und Manager, sondern auch als ganz besonders kreative und hilfreiche Persönlichkeit erlebt.

Hans Götschhofer wurde am 11. November 1947 in Linz geboren, wo er auch die Volks- und Hauptschule besuchte. Danach besuchte er die Fachschule und die HTL Maschinenbau und Fahrzeugtechnik in Mödling, die er 1968 mit der Matura abschloss. Er studierte von 1969 - 1973 Betriebswirtschaft an der Hochschule für Welthandel und trat 1973 in die Betriebswirtschaftsabteilung der VOEST ALPINE ein, wo er in den Bereichen Kostenträgerplanung, Kostenplanung und -kontrolle tätig war.

1979 übernahm er die Leitung der Abteilung Ergebnisplanung und -kontrolle, 1983 die Abteilung Betriebs-

wirtschaft und 1987 wurde er Leiter der Controlling Hütte Linz und Abteilungsdirektor in der VOEST - ALPINE Stahl GmbH. 1989 übersiedelte er in die VOEST - ALPINE AG als Direktor der Bereichsplanung und -koordination und war ab Herbst 1991 Leiter der Konzernstrategie und -planung der VOEST - ALPINE AG. Nach Verschmelzung der VOEST - ALPINE AG mit Austrian Industries war er Bereichsleiter für "Controlling Qualitätsstahl/ Handel“ in der AUSTRIAN INDUSTRIES AG. Von 1994 bis 1999 wirkte er als Prokurist und Leiter der Konzernplanung der voestalpine AG. Seit 1996, bis zu seiner Pensionierung im März 2010, war er zusätzlich für die interne Revision der voestalpine AG verantwortlich. Ab Oktober 1999 führte er die Unternehmensentwicklung der voestalpine Stahl $\mathrm{GmbH}$.

Er leitete seit der Gründung bis zu seinem Ruhestand den gemeinsamen Betriebswirtschaftsausschuss von ASMET und VDEh, diesen für die deutsche und die österreichische Werkstoffindustrie sehr wichtigen Fachausschuss.

Ein wesentlicher Schwerpunkt seiner Arbeit war die Einführung von Managementsystemen in der Hüttenindustrie. Der Aufbau eines systematischen Wissensaustauschs zwischen Universitäten und Mitgliedswerken war ein wesentlicher Beitrag zum Change Management und zur erfolgreichen Weiterentwicklung der Stahlindustrie.

Unsere besondere Anteilnahme gilt seiner Frau Margarethe, seiner Tochter und seinen Enkelkindern.

Wir sind dankbar, dass wir einen Teil unseres Weges mit ihm gemeinsam gehen durften. Wir alle werden Hans Götschhofer aufrichtig vermissen und ihm ein würdiges Andenken bewahren.

Ein stilles Glück auf!

Josef Neuwirth Bruno Hribernik 